\title{
Estudo epidemiológico da fibromialgia em ambulatório municipal de reumatologia no Estado do Tocantins
}

\section{Epidemiological study of fibromyalgia in a municipal ambulatory of rheumatology in the State of Tocantins}

Edson Pedroza dos Santos Junior ${ }^{1}$, Poliana Guerino Marson², Victor Rodrigues Nepomuceno ${ }^{3}$

\section{RESUMO}

A Fibromialgia é caracterizada por dor crônica, geralmente associada a fadiga, distúrbios do sono e sintomas cognitivos. A prevalência no Brasil é elevada, acometendo principalmente mulheres. $O$ objetivo do trabalho foi descrever o perfil epidemiológico e determinar a incapacidade para tarefas em pacientes com fibromialgia. Estudo transversal realizado por meio de aplicação do Questionário de Impacto da FM (QIF-Br) e análise de questionários elaborados pelo grupo EpiFibro, aplicados a pacientes que atenderam os critérios diagnósticos do ACR de 2010, em ambulatório municipal de reumatologia no estado do Tocantins, no período de junho a novembro de 2018. Foram analisados 52 pacientes do sexo feminino, com idades entre 26 a 74 anos, casadas, com renda familiar entre um e três salários mínimos, desempregadas ou aposentadas e nível de escolaridade médio e superior. A maioria das pacientes apresentaram limitações para realizar atividades físicas diárias, dores (moderada/grave) e vários sintomas que corroboram com a incapacidade causada pela doença, resultando em elevados valores na escala analógica visual, IDG e no escore total do QIF (> 50), em cerca de $80,76 \%$ das pacientes. Os resultados dos parâmetros clínicos nos casos avaliados têm scores elevados, demonstrando o impacto negativo na qualidade de vida dessas pacientes.

Palavras-chave: Fibromialgia; Epidemiologia; Critério diagnóstico

\section{ABSTRACT}

Fibromyalgia is characterized by chronic pain, usually associated with fatigue, sleep disorders and cognitive symptoms. The prevalence in Brazil is high, affecting mainly women. The aim of the study was to describe the epidemiological profile and determine the inability to perform tasks in patients with fibromyalgia. Cross-sectional study conducted through the application of the FM Impact Questionnaire (QIF-Br) and analysis of questionnaires prepared by the EpiFibro group, applied to patients who met the 2010 ACR diagnostic criteria, in a municipal rheumatology clinic in the state of Tocantins, from June to November 2018. 52 female patients, aged 26 to 74 years, married, with family income between one and three minimum wages, unemployed or retired, and average and higher education level were analyzed. Most patients had limitations to perform daily physical activities, pain (moderate / severe) and several symptoms that corroborate the disability caused by the disease, resulting in high values on the visual analog scale, IDG and in the total QIF score (>50), in about $80.76 \%$ of the patients. The results of clinical parameters in the evaluated cases have high scores, demonstrating the negative impact on the quality of life of these patients.

${ }^{2}$ Farmacêutica-Bioquímica,Doutora em Biotecnologia, Docente do Programa de Pós-graduação em Ciências da Saúde, Universidade Federal do Tocantins - UFT.

Fisioterapeuta, Doutor em Ciências, Docente do Programa de Pósgraduação em Ciências da Saúde,

Keywords: Fibromyalgia; Epidemiology; Diagnostic criteria 


\section{INTRODUCÃO}

A Fibromialgia (FM) é uma condição clínica caracterizada por dor crônica generalizada, normalmente associada a fadiga, distúrbios do sono e sintomas cognitivos. Sua prevalência foi estimada entre $2 \%$ a $5 \%$ da população geral (MARTINEZ-LAVIN, 2012), sendo no Brasil, de aproximadamente $2,5 \%$, semelhante a outros países (RESENDE et al., 2013). Seu acometimento é maior em mulheres quando comparada a homens, e raramente afetando crianças. Com a utilização dos critérios diagnósticos modificados do American College of Rheumatology (ACR - 2010), foi observado uma diminuição na incidência entre mulheres e homens de 8:1 para 4:1, quando comparado ao uso dos critérios diagnósticos anteriormente utilizados. Isso pode ser justificado devido ao fato desses critérios modificados permitirem a identificação de FM sem verificação dos pontos sensíveis à palpação (WOLFE et al., 2010).

Acredita-se que o início da FM frequentemente está associado a algum agente desencadeante que se comporta como "fator gatilho" e aciona a síndrome. Dentre esses agentes podemos citar: trauma físico ou emocional, doenças infecciosas, perdas prolongadas do sono, modificações hormonais, mudanças climáticas e doenças autoimunes (GOLDENBERG, 2015).

Os diversos sintomas observados na FM, que podem dificultar a avaliação e o diagnóstico, são: cefaleia, fadiga, dor e fraqueza muscular, problemas de memória, parestesias, transtorno depressivo, ansiedade, alteração do trânsito intestinal, dismenorreia, edema subjetivo, síndrome uretral, dor pélvica e síndrome seca, sintomas esses atribuídos à disfunção do sistema nervoso autonômico (BROSSEAU et al., 2008; KULSHRESHTHA e DEEPAK, 2013). Outros sintomas citados pela literatura como sendo frequentes para essa patologia são: palpitação, tontura, sensação de inchaço, dor torácica, cefaleia crônica, irritabilidade, zumbido, epigastralgia, dispneia, enjoo, dificuldade de digestão, fenômeno de Raynauld e cólon irritável (HELFENSTEIN Jr e FELDMAN, 2002; RIBERTO e BATTISTELLA, 2002).

A ausência de testes laboratoriais ou de imagem torna um desafio o diagnóstico da doença, especialmente em contexto de atenção primária, onde pacientes que buscam atendimento apresentam quadros de somatização crônica que podem confundir o processo diagnóstico, tornando-o difícil e muitas vezes demorado. Portanto, é uma 
doença cujo diagnóstico é basicamente clínico, de forma que os testes complementares se destinam a excluir outras hipóteses (WOLFE et al., 2010).

Em 2010, o American College of Rheumatology (ACR) apresentou os Critérios Diagnósticos Preliminares para a FM e aboliram a contagem de pontos sensíveis à palpação, enfatizando a associação de fadiga, distúrbios do sono, distúrbios cognitivos e sintomas somáticos associados à dor crônica generalizada. Esses critérios estabelecem dois escores - um índice de dor generalizada (IDG / WPI) composto por 19 áreas potencialmente dolorosas a serem identificadas pelos pacientes durante avaliação física, e um índice de gravidade dos sintomas (EGS/SSI) que resulta da soma dos escores de fadiga, distúrbios do sono, distúrbios cognitivos e sintomas somáticos ( 0 a 3 cada), sendo que o escore total varia de 0 a 12 (WOLFE et al., 2010). Em 2011, o item sintomas somáticos foi modificado, o que possibilitou a criação de uma versão autorrelatada, a fim de ser usada em estudos epidemiológicos (WOLFE et al., 2011; HEYMANN et al., 2017). As medidas de seguimento para os pacientes com FM normalmente são escalas analógicas de sintomas, como por exemplo para a dor, e questionários de qualidade de vida ou o impacto que a doença tem na vida dos pacientes, com o QIF e sua forma revisada, cujas versões foram traduzidas e validadas para o Brasil (PAIVA et al., 2013; LUPI et al., 2017). A soma de IDG e EGS, que varia de 0 a 31, é chamada Polysymptomatic Distress Scale (PDS) e também pode ser usada para seguimento clínico (WOLFE, et al., 2015).

A fim de analisar a epidemiologia e as comorbidades da FM na população brasileira, almejando alcançar a eficácia da conduta diagnóstica e terapêutica (REZENDE et al., 2013), introduzindo medidas para encurtar o tempo de efetivação diagnóstica, aprimorar o diagnóstico, tratar mais precocemente, adequar melhor o tratamento e melhorar a qualidade de vida dos pacientes com FM, foi criado o Estudo Epidemiológico de Fibromialgia no Brasil (EpiFibro) (MARTINEZ et al., 2017).

A presente pesquisa torna-se relevante para agregar aos dados nacionais, particularidades da região norte, pois a maior parte das informações existentes referem-se às regiões sul e sudeste do país. Dessa forma, o objetivo dessa pesquisa foi identificar pacientes que se enquadram aos critérios diagnóstico da comissão do ACR para FM, traçar seu perfil epidemiológico, assim como analisar a incapacidade para tarefas diárias, em um centro de referência em reumatologia na região norte do Brasil, estimula maior 
conhecimento sobre a realidade local da doença, a educação continuada de profissionais de saúde, proporcionando melhor prognóstico, diagnóstico prévio e condução clínica desses pacientes.

\section{MATERIAIS E METODOS}

Trata-se de um estudo transversal, realizado por meio de aplicação de Questionário de Impacto da FM (QIF-Br) e análise de questionários padrão EpiFibro, à pacientes portadores de FM, durante a consulta no serviço de reumatologia do Ambulatório Municipal de Atenção à Saúde (AMAS), em Palmas - Tocantins, onde foram entrevistados 463 pacientes, com idade entre 18 e 80 anos, do sexo feminino, com provável diagnóstico de FM, no período de 01 junho de 2018 a 30 novembro de 2018. Foram selecionados 52 pacientes que atenderam aos critérios diagnósticos do ACR de 2010 modificados. Foram excluídos os pacientes portadores de algum déficit cognitivo, doenças reumáticas autoimunes, bem como os que não consentiram em participar da pesquisa.

As pacientes selecionadas foram convidadas a participar da pesquisa no momento da consulta com o reumatologista. Após serem diagnosticadas com FM, foram convidadas a participar da pesquisa e responder o questionário padronizado pelo programa EpiFibro. No aceite em participar da pesquisa, antes da realização de qualquer procedimento, foi apresentado e explicado o Termo de Consentimento Livre e Esclarecido (TCLE) para assim, iniciar a coleta das informações por meio dos instrumentos.

A aquisição dos dados das pacientes se deu por meio de entrevista, e as informações foram coletas por meio de Anamnese, Escala Visual Analógica de dor (EVA), Questionário de Impacto da Fibromialgia (QIF-Br) e Questionários padrão EpiFibro. Os dados foram computados em planilha eletrônica, a partir da qual foram calculadas as medidas estatísticas: dados epidemiológicos gerais foram apresentados como proporções das diferentes respostas observados a cada item do questionário. Dentre os cálculos utilizados, foram avaliadas médias, desvio padrão e coeficientes de correlação ( $r$ ) Pearson.

- Neste estudo foram respeitadas as Diretrizes e Normas da Resolução nำ466/212, do Conselho Nacional de Saúde, tendo sido aprovado pelo Comitê de Ética em Pesquisa 
do Instituto Federal do Tocantins (CEP/IFTO), sob no CAAE 88208818.4.0000.8111, em 09 de maio de 2018.

\section{RESULTADOS}

Dos 463 pacientes atendidos no ambulatório de reumatologia do AMAS, no período, pelo menos 30\% (139) foram encaminhados por médicos da atenção básica ou especializada com quadro de dores musculares e articulares (difusas e crônicas). Um total de 77 pacientes foram encaminhados com suspeita de FM, sendo que apenas 41 foram confirmados com a doença. Quanto aos demais 62 encaminhamentos com quadro de dor crônica, apenas 11 pacientes atenderam os critérios diagnóstico para FM, totalizando 52 pacientes que foram incluídos no estudo.

$\mathrm{Na}$ Tabela 1 estão demonstradas as características físicas das pacientes do estudo. A faixa etária foi de 26 a 74 anos, com peso variando de 43 a $103 \mathrm{~kg}$ e a estatura de 1,40 a 1,79 cm. O índice de massa corpórea (IMC) foi elevado nas pacientes do estudo.

Tabela 1 - Média e desvio padrão das características físicas das pacientes do estudo.

\begin{tabular}{cc}
\hline Variáveis & $\begin{array}{c}\mathbf{N}^{\circ} \text { (Média e desvio } \\
\text { padrão) }\end{array}$ \\
\hline Idade (anos) & $46,13 \pm 10,01$ \\
Peso $(\mathrm{Kg})$ & $68,72 \pm 12,70$ \\
Estatura $(\mathrm{cm})$ & $1,57 \pm 0,07$ \\
IMC & $27,80 \pm 5,38$
\end{tabular}

Fonte: Elaborado pelo autor.

A Tabela 2 apresenta as características socioeducacionais e hábitos de vida da amostra.

A prática regular de exercício físico, em diferentes modalidades, foi relatada durante a entrevista na primeira consulta por $52 \%$ das participantes, sendo a caminhada a modalidade mais praticada. Dessas pacientes, 44\% realizavam a atividade física entre 4 a 7 dias por semana, e 56\% relataram praticar de 2 a 3 dias por semana. A maioria das pacientes relataram não ser tabagistas, e seis faziam uso esporádico de bebida alcoólica, assim, apenas uma relatou uso associado de tabaco e bebida alcoólica. 
Tabela 2 - Distribuição de frequência de características socioeducacionais e clínicas das pacientes do estudo $(n=52)$.

\begin{tabular}{|c|c|c|}
\hline Variáveis & & $\mathbf{N}^{\circ}(\%)$ \\
\hline \multirow[t]{4}{*}{ Estado civil } & Casado & $30(57,5 \%)$ \\
\hline & Solteiro & $11(21 \%)$ \\
\hline & Divorciado & $07(13,5 \%)$ \\
\hline & Viúvo & $04(8 \%)$ \\
\hline \multirow[t]{4}{*}{ Escolaridade } & Fundamental completo & $07(13,5 \%)$ \\
\hline & Fundamental incompleto & $06(11,5 \%)$ \\
\hline & Ensino médio & 24 (46\%) \\
\hline & Superior & $15(29 \%)$ \\
\hline \multirow[t]{3}{*}{ Trabalho } & Empregado / Autônomo & 27 (52\%) \\
\hline & Não empregado & $10(19 \%)$ \\
\hline & Do lar / Aposentado & $15(29 \%)$ \\
\hline Renda mensal & $<1$ & $13(25 \%)$ \\
\hline \multirow[t]{2}{*}{ (n Salário) } & 1 a 3 & $33(63,5 \%)$ \\
\hline & 4 a 6 & $06(11,5 \%)$ \\
\hline \multirow[t]{2}{*}{ Atividade física } & Realizava & $27(52 \%)$ \\
\hline & Não realizava & $25(48 \%)$ \\
\hline \multirow[t]{2}{*}{ Tabagismo } & Sim & $04(7,7 \%)$ \\
\hline & Não & $48(92,3 \%)$ \\
\hline
\end{tabular}

Fonte: Elaborado pelo autor.

Foi realizada comparação entre as participantes que praticavam e aquelas que não praticaram atividade física em relação aos parâmetros de EVA, QIF e PDS, onde as médias obtidas que avaliam a qualidade de vida foram bem próximas, não mostrando superioridade quanto a prática de atividade física (Tabela 3). 
Tabela 3 - Média padrão dos parâmetros clínicos dos pacientes da pesquisa relacionados à prática de atividade física

\begin{tabular}{lll}
\hline Parâmetro & Sim & Não \\
\hline EVA & 7,0 & 6,4 \\
PDS & 19,8 & 20,6 \\
QIF & 62,5 & 67,5 \\
\hline
\end{tabular}

Fonte: Elaborado pelo autor

Isso pode ser justificado pelos resultados dessa pesquisa, onde parâmetros avaliados com a média dos scores se apresentaram bem próximos, quando foram correlacionados pacientes que realizavam atividade física na frequência 2 a 3 vezes na semana (56\%) com médias de EVA $(6,3)$, QIF $(66,9)$, PDS $(19,5)$, e pacientes que realizavam atividade física na frequência 4 a 7 vezes na semana (44\%) com médias de EVA $(5,6)$, QIF $(57,7)$, PDS $(20,6)$. Deve ser levado em consideração que os resultados são baseados na aplicação do questionário apenas na primeira consulta e que as pacientes não foram separadas por modalidade esportiva, podendo os resultados da presente pesquisa apresenta limitações quando comparados a pesquisas onde os participantes foram expostos a uma única modalidade e/ou comparação entre duas modalidades esportivas e acompanhamento médico.

No que tange ao tempo de dor e diagnóstico, $84,6 \%$ das pacientes estudadas relataram sentir dor a mais de 4 anos. No entanto, $34,6 \%$ foram diagnosticadas a menos de quatro anos e o tempo para o diagnóstico chegou a ultrapassar dois anos.

Destaca-se que $76,9 \%$ das pacientes estudadas apresentavam mais de uma patologia clínica, além da FM, sendo elas: transtorno comportamental, arritmia, osteoartrite, lombalgia, litíase renal, glaucoma, diabetes mellitus, endometriose e predominância da hipertensão arterial sistêmica (HAS).

Com relação ao IDG observado nas pacientes, cerca de $67 \%$ delas relataram dores em mais de 9 áreas do corpo, havendo relatos de pacientes com até 18 áreas, acompanhado da EGS com escore $\geq 9$ em aproximadamente 60\%. Quanto a avaliação EVA, foi observado que $34,5 \%$ das pacientes apresentaram dores intensas, enquanto $56 \%$ alegam dores moderadas e apenas $9,5 \%$ sofrem com dores leves. Apesar de não utilizado nesta pesquisa, quando analisado pelos critérios diagnósticos antigos (ACR de 
1990), apenas $63,46 \%$ das pacientes estudadas se enquadrariam no perfil da doença, demonstrando menor sensibilidade aos antigos critérios diagnósticos do ACR de 1990.

Dentre as pacientes estudadas, observou-se dificuldades ao avaliar a capacidade física em atividades comuns como: escovar os dentes, pentear o cabelo, caminhar continuamente por 20 minutos, realizar atividades domésticas de limpeza, subir escadas, ficar sentado continuamente por 45 minutos e, principalmente, erguer ou carregar sacolas de compras.

Indícios de diminuição da qualidade do sono também foi observado em número elevado de entrevistadas. O escore total do QIF, que varia de 0 a 100, no presente estudo apresentou-se alto (acima de 50 pontos) em cerca de $80,7 \%$ dos pacientes, indicando o alto índice (severo) de incapacidade para atividades diárias.

\section{DISCUSSÃO}

$\mathrm{Na}$ presente pesquisa, apesar de terem sido selecionadas apenas mulheres com FM, não foram observados homens que atendessem os critérios diagnósticos para a doença, confirmando assim a predominância do acometimento do sexo feminino, compatível com as descrições da literatura (WOLFE et al., 2010; MARQUES et al.; 2017).

A faixa etária predominante foi entre 30 a 55 anos, sendo a média 46 anos, resultados semelhantes foram encontrados em outras pesquisas no país (MARTINEZ et al., 2013; GEQUELIM et al., 2013).

Apesar de uma parte significativa das pacientes estarem com situação de sobrepeso e obesidade, identificado pelo elevado IMC, não foi possível identificar se a obesidade é a causa ou consequência do problema. Discussão semelhante tem sido abordada em trabalho correlacionando FM e obesidade (ARAÚJO \& CRISPIM, 2014).

Os pacientes frequentemente referem indisposição, rigidez e fadiga muscular, além de dor musculoesquelética contínua e difusa, sendo cada um desses distúrbios as causas e, concomitantemente, as consequências da piora do quadro sintomático, da incapacidade para o trabalho e da atividade física observada nas pacientes com FM (SALVADOR, SILVA e ZIRBES, 2005; MARTINS et al., 2011). Todas essas queixas foram referidas como frequentes nas pacientes do presente estudo, além de outros sintomas, como: distúrbios de sono, cefaleia persistente e transtorno de comportamento. Pacientes com FM tendem a ter seus sintomas agravados quando apresentam distúrbios 
psicológicos associados, por meio de possíveis mecanismos: reduzindo a tolerância à dor, impossibilitando a adesão ao tratamento e diminuindo a funcionalidade física. A depressão está relacionada a uma piora da funcionalidade social e emocional, da saúde mental e da percepção da saúde em geral (BERBER, KUPERK e BERBER, 2005).

A hipertensão arterial sistêmica (HAS) foi a patologia mais encontrada associada a FM em pelo menos $30 \%$ das entrevistadas. Outras patologias observadas foram transtorno comportamental, arritmia, osteoartrite, lombalgia, litíase renal, glaucoma, diabetes mellitus e endometriose. Gequelim e colaboradores, em 2012, encontraram resultados próximos em relação à HAS, em $45,2 \%$ de seus pacientes, seguido de hipotireoidismo e outras doenças cardiovasculares. Apesar de estudos demonstrarem a associação comum de FM a outras patologias (CASTRO, KITANISHI e SKARE, 2011), também deve ser levado em consideração, o fato dessa doença acometer mulheres com idade acima de 40 anos (FRAGA et al., 2012; DIAS et al., 2017). Em recente estudo realizado nos Estados Unidos, foram avaliadas as comorbidades da fibromialgia nos últimos 10 anos, e dentre as principais doenças associadas à FM, destacaram-se as psiquiátricas, seguidas dos distúrbios do sono (MELO, MADRUGA e TORRO, 2020). A associação da FM a comorbidades aumenta o sofrimento e piora a qualidade de vida destes indivíduos (HEYMANN et al., 2010)

Quando avaliada a prática de exercícios físicos, foi possível observar que paciente que praticavam baixa frequência demonstrou resultados semelhantes aos pacientes que realizavam de moderada à alta frequência, apesar de não ter sido estimada a intensidade e modalidades praticadas, estas parecem ter influenciado o resultado do estudo em não demonstrar melhora da dor e qualidade de vida nas pacientes. Há uma dificuldade em mensurar a intensidade da dor e sua relação com a atividade física. Ademais, o IMC da maioria das pacientes está elevado, o que pode sugerir que a quantidade de atividade física, não está adequada, indicando sedentarismo. A escolha da modalidade física, sua frequência e intensidade podem causar reações diferentes nas pacientes, o que pode trazer resultados subjetivos e interferir nos resultados obtidos (CONTE et al., 2018).

Foi observado que limitações quanto a demora do diagnóstico, realização de atividade física e dificuldade de acesso a medicamentos parecem ser alguns dos principais motivos para justificar o maior número de relatos de dores moderadas a intensa. 
Os resultados levantados com aplicação do QIF indicam uma diminuição da capacidade física, relacionada a um maior nível de dor, piorando gravemente a qualidade de vida das mulheres que sofrem com hipersensibilidade à dor, corroborando com os achados de Lorena e colaboradores (2016). Foi também observado indícios de diminuição da qualidade do sono, nas pacientes entrevistas. Estudos demonstram que quando a qualidade do sono é beneficiada, também há melhora da dor e da fadiga muscular, em portadores de FM (MARQUES et al., 2006).

Houve uma correlação positiva (Pearson), porém baixa $(r=0,39)$, entre os parâmetros QIF e PDS. Essa correlação pode ser explicada pela coleta de dados de pacientes em diferentes estágios da doença, onde participaram aqueles em período latente, assim como os que se encontravam em plena atividade da doença. A escolha dos dois parâmetros se deu por sua praticidade em ser aplicado por médicos não reumatologistas, principalmente nas unidades básicas de saúde para auxílio e acompanhamento de pacientes com FM. O QIF evidenciou resultados confiáveis na amostra estudada, demonstrando ser uma ferramenta útil que pode ser utilizada em pesquisa e prática clínica de seguimento dos pacientes. Porém o PDS, criado posteriormente, requer mais estudos que o consolidem como potencial parâmetro no monitoramento clínico (MARQUES, 2006). Segundo Marques (2006) e Martinez et al. (2017) melhores resultados têm sido descritos quando correlacionados os mesmos instrumentos em pesquisas, onde seja realizada a observação e o seguimento de pacientes com FM.

\section{CONSIDERACÓES FINAIS}

É indispensável que a FM, com sua alta complexidade e grau de comprometimento, associada às limitações e ao baixo desempenho nas atividades laborais, seja entendida, pois é de suma importância que seus sinais e sintomas sejam identificados, visando o diagnóstico precoce e o devido tratamento.

Os resultados dos parâmetros clínicos observados demonstraram que os casos avaliados têm scores de impacto de vida muito elevados, classificando como grave os achados. O fato do centro de coleta de dados pertencer ao nível secundário do sistema único de saúde pode ser uma justificativa, pois nesses locais observa-se limitações de acesso a medicamentos, assim como a baixa periodicidade junto à equipe multiprofissional pelas pacientes com FM. 
Podemos identificar neste estudo que os pacientes encaminhados por profissionais não reumatologistas, apresentaram deficiência na celeridade de conduta diagnóstica e terapêutica. Isso foi revelado pelo tempo de dor difusa até o diagnóstico definitivo, de forma que tal situação merece atenção por parte de todos os profissionais da área da saúde para disseminar informações a respeito da FM.

Os dados levantados nesta pesquisa mostraram semelhanças às pesquisas realizadas em outras regiões do país, não apresentando particularidades relevantes que distinguisse alguma característica clínica específica da região estudada e que pudesse influenciar de forma negativa as manifestações da doença.

Novos estudos devem ser estimulados para que a adoção de estratégias de atenção à saúde para que o portador de FM receba mais atenção e cuidados diferenciados.

\section{REFERÉNCIAS}

ARAÚJO, TA.; CRISPIM, CA. Relação entre padrão de sono e obesidade em mulheres com Fibromialgia. 2014. 76 f. Dissertação (Mestrado em Ciência da saúde) Faculdade de Medicina, Universidade Federal de Uberlândia, 2014.

BERBER, J. S. S.; KUPEK, E.; BERBER, S. C. Prevalência de depressão e sua relação com a qualidade de vida em pacientes com síndrome da fibromialgia. Rev Bras Reumatol, 45(2), 2005, p. 47-54.

BROSSEAU, L. et al., Ottawa Panel Evidence-Based Clinical Practice Guidelines for Strengthening Exercises in the Management of Fibromyalgia: Part 2. Physical Therapy, New York, v. 88, n. 7, 2008, p. 873-886.

CASTRO, A. A., KITANISHI, L. K., \& Skare, T. L. (2011). Fibromialgia no homem e na mulher: estudo sobre semelhanças e diferenças de género. Arquivos Catarinenses Medicina, 40(2), 63-69.

CONTE, M. et. al. Fibromialgia: atividade física, depressão e qualidade de vida. Medicina (Ribeirão Preto. Online), v. 51, n. 4, p. 281-290, 2018.

DIAS, C. Z. et al. Perfil dos usuários com doenças reumáticas e fatores associados à qualidade de vida no sistema único de saúde, Brasil. Rev Med Minas Gerais, v. 27, e1901, p. 1-7, 2017.

FRAGA, B.P.; SANTOS, E.B.; FARIAS NETO, J.P.; MACIEIRA, J.C.; QUINTANS, L.J.JR.; ONOFRE, A.S.; DE SANTANA, J.M.; MARTINS-FILHO, P.R.; BONJARDIM, L.R. Signs and symptoms of temporomandibular dysfunction in fibromyalgic patients. $\mathbf{J}$ Craniofac Surg, 2012 Mar;23(2):615-8. doi: 10.1097/SCS.0b013e31824cd81a. 
GEQUELIM, G. C. et. al. Estudo clínico-epidemiológico de fibromialgia em hospital universitário do sul do Brasil. Rev. Méd, Paraná, Curitiba. 2012; 70(2):7-12.

GOLDENBERG, E. O coração sente, o corpo dói: como reconhecer e tratar a fibromialgia. 7 ed, São Paulo: Atheneu, 2015.

HELFENSTEIN JR, M.; FELDMAN, D. Síndrome da fibromialgia: características clínicas e associação com outras síndromes disfuncionais. Rev Bras Reumatol, 42, 2002, p. 7-14.

HEYMANN, R. E. et al., Novas diretrizes para o diagnóstico da fibromialgia. Rev Bras Reumatol, São Paulo, v. 57 (S 2), 2017, p. 467-476.

HEYMANN, R. E., PAIVA, E. D. S., HELFENSTEIN, M. Jr., POLLAK, D. F., MARTINEZ, J. E., PROVENZA, J. R., \& LAGE, L. V. (2010). Consenso brasileiro do tratamento da fibromialgia. Revista Brasileira de Reumatologia, 50(1), 56-66. Acesso em:

http://www.scielo.br/pdf/rbr/v50n1/v50n1a06.

KULSHRESHTHA, P.; DEEPAK, K. K. Autonomic nervous system profile in fibromyalgia patients and its modulation by exercise: a mini review. Clin Physiol Funct Imaging, Orford, v. 33, 2013, p. 83-91.

LORENA, S. B. et al., Avaliação de dor e qualidade de vida de pacientes com fibromialgia. Rev Dor, São Paulo, 17(1), jan-mar, 2016, p. 8-11.

LUPI, J. B. et al., Brazilian Portuguese version of the Revised Fibromyalgia Impact Questionnaire (FIQR-Br): cross-cultural validation, reliability, and construct and structural validation. Journal Disability and Rehabilitation, Oxfordshire, v. 39, n. 16, ago. 2017, p. 1650-1663.

MARQUES, A. et al., Validação da versão brasileira do Fibromyalgia Impact Questionnaire (FIQ). Rev Bras Reumatol, 46, 2006, p. 24-31.

MARQUES, A. P. et al., A prevalência de fibromialgia: atualização da revisão de literatura. Rev Bras Reumatol, São Paulo, v. 57, n. 4, jul-ago. 2017, p. 356-363.

MARTINEZ, J. E. et al. EpiFibro. (Registro Brasileiro de Fibromialgia): dados sobre a classificação do ACR e preenchimento dos critérios diagnósticos preliminares e avaliação de seguimento. Rev Bras Reumatol, São Paulo, v. 57, n. 2, mar-abr 2017, p.129-133.

MARTINEZ-LAVIN, M. Fibromyalgia when distress becomes(un) sympathetic pain. Pain Res Treat, 981565, 2012.

MARTINS, M. R. et al., Uso de questionários para avaliar a multidimensionalidade e a qualidade de vida do fibromiálgico. Rev Bras Reumatol, 52(1), 2011, p. 16-26.

MELO, G., MADRUGA, M., TORRO, N. Análise das comorbidades associadas à fibromialgia: uma revisão bibliométrica. Psic., Saúde \& Doenças, 21(2), ago 2020, p. 395-403. Acesso em: http://dx.doi.org/10.15309/20psd210214. 
PAIVA, E. S. et al., A Brazilian Portuguese version of the Revised Fibromyalgia Impact Questionnaire (FIQR): avalidation study. Clin Rheum, 32, 2013, p. 1199-1206.

REZENDE, M. C. et al., EpiFibro (Registro Brasileiro de Fibromialgia): um banco de dados nacional sobre a síndrome da fibromialgia - análise inicial de 500 mulheres. Rev Bras Reumatol, São Paulo, v. 53, n. 5, mar 2013, p. 382-387.

RIBERTO, M.; BATTISTELLA, L. R. Comorbidades em Fibromialgia. Rev Bras Reumatol, 42, 2002, p. 1-7.

SALVADOR, J. P.; SILVA, Q. F.; ZIRBES, M. C. Hidrocinesioterapia no tratamento de mulheres com fibromialgia: estudo de caso. Fisioter Pesq, 11(1), 2005, p. 27-36.

WOLFE F. et al., The American College of Rheumatology preliminary diagnostic criteria for fibromyalgia and measurement of symptom severity. Arthritis Care Res, (Hoboken), 62(5), 2010, p. 600-610.

WOLFE, F. et al., Fibromyalgia criteria and severity scales for clinical and epidemiological studies: a modification of the ACR Preliminary Diagnostic Criteria for Fibromyalgia. J Rheumatol, 38(6), 2011, p. 1113-1122.

WOLFE, F. et al., The use of polysymptomatic distress categories in the evaluation of fibromyalgia (FM) and FM severity. J Rheumatol, 2015, 42, p. 1494-1501. 